\title{
Three-dimensional co-culture of mesenchymal stromal cells and differentiated osteoblasts on human bio-derived bone scaffolds supports active multi-lineage hematopoiesis in vitro: Functional implication of the biomimetic HSC niche
}

\author{
XIAOBING HUANG, BIAO ZHU, XIAODONG WANG, RONG XIAO and CHUNSEN WANG \\ Department of Hematology, Sichuan Academy of Medical Sciences and \\ Sichuan Provincial People's Hospital, Chengdu, Sichuan 610072, P.R. China
}

Received September 22, 2015; Accepted June 29, 2016

DOI: $10.3892 /$ ijmm.2016.2712

\begin{abstract}
Recent studies have indicated that the hematopoietic stem/progenitor cell (HSPC) niche, consisting of two major crucial components, namely osteoblasts (OBs) and mesenchymal stromal cells (MSCs), is responsible for the fate of HSPCs. Thus, closely mimicking the HSPC niche ex vivo may be an efficient strategy with which to develop new culture strategies to specifically regulate the balance between HSPC self-renewal and proliferation. The aim of this study was to establish a novel HSPC three-dimensional culture system by co-culturing bone marrow-derived MSCs and OBs differentiated from MSCs without any cytokines as feeder cells and applying bio-derived bone from human femoral metaphyseal portion as the scaffold. Scanning electron microscopy revealed the excellent biocompatibility of bio-derived bone with bone marrow-derived MSCs and OBs differentiated from MSCs. Western blot analysis revealed that many cytokines, which play key roles in HSPC regulation, were comprehensively secreted, while ELISA revealed that extracellular matrix molecules were also highly expressed. Hoechst 33342/propidium iodide fluorescence staining proved that our system could be used to supply a long-term culture of HSPCs. Flow cytometric analysis and qPCR of p21 expression demonstrated that our system significantly promoted the self-renewal and ex vivo expansion of HSPCs. Colony-forming unit (CFU) and long-term cultureinitiating cell (LTC-IC) assays confirmed that our system has the ability for both the expansion of $\mathrm{CD} 34^{+}$hematopoietic stem cells (HPCs) and the maintenance of a primitive cell subpopulation of HSCs. The severe-combined immunodeficient mouse repopulating cell assay revealed the promoting effects of our
\end{abstract}

Correspondence to: Dr Chunsen Wang, Department of Hematology, Sichuan Academy of Medical Sciences and Sichuan Provincial People's Hospital, 32 West Second Section First Ring Road, Chengdu, Sichuan 610072, P.R. China

E-mail: wangsjsjsdq2@163.com

Key words: osteoblasts, mesenchymal stromal cells, hematopoietic stem/progenitor cell niche, three-dimensional culture system system on the expansion of long-term primitive transplantable HSCs. In conclusion, our system may be a more comprehensive and balanced system which not only promotes the self-renewal and ex vivo expansion of HSPCs, but also maintains primitive HPCs with superior phenotypic and functional attributes.

\section{Introduction}

Hematopoietic stem cells (HSCs) are defined by their ability to generate all cells of the hematopoietic system. The stem cell niche provides a specific microenvironment for HSCs to reside, and is responsible for their fate in terms of quiescence, selfrenewal and differentiation (1). Recent studies have clarified the role of the marrow microenvironment in the pathogenesis of hematologic tumors. It has been reported that the identification of target molecules can be exploited to eradicate the leukemic stem cells from the niche $(2,3)$. Modern hematopoietic stem/ progenitor cell (HSPCs) culture systems that closely mimic marrow physiology, can provide an experimental tool with which to understand the niche-mediated regulation of HSCs, under both physiological and diseased conditions (4). The latter may potentially help to design and develop novel therapeutic strategies to target the HSC niche. In addition, during the past three decades, HSC transplantation has become a well-established treatment for hematologic malignancies and non-malignant disorders. To improve the clinical outcome of autologous and allogeneic HSC transplantation, many study groups are focusing on the ex vivo expansion of HSCs, particularly for those cases in which the graft is of limited size, such as HSCs from cord blood (5-7). However, the expansion of HSCs in vitro is difficult to be achieved due to known stem cell characteristics. Although the contribution of conventional hematopoietic culture systems to the knowledge of human HSC biology is unquestionable, these existing HSC culture systems cannot meet the requirements of the clinical application. Therefore it is necessary to constantly improve ex vivo experimental systems to closely resemble their in vivo counterparts.

HSCs are regulated by intrinsic mechanisms and extrinsic signals mediated via specialized microenvironments known as 'niches'. The self-renewal and differentiation ability of HSCs are regulated by two major elements: endothelial and vascular 
regulatory elements. In addition, the osteoblastic niche localized at the inner surface of the bone cavity has been recognized as the main regulator of HSC fate and serves as a reservoir for long-term HSC storage in a quiescent state $(8,9)$. The deletion of the gene Dicer expressed specifically in osteo-progenitors and immature osteoblasts (OBs), has been shown to affect hematopoiesis, indicating the involvement of a precise cell group within osteo-lineage cells in HSC maintenance (10). Taichman et al demonstrated that the in vitro culture of human bone marrow CD $34^{+}$cells with human OBs supported a 3-4-fold expansion of long-term culture-initiating cells in vitro (11). On the other hand, the vascular niche predominantly consists of the bone marrow sinusoidal endothelial cells, which are a part of the vascular system, and perivascular cells which surround the bone marrow vasculature, known as mesenchymal stem cells or mesenchymal stromal cells (MSCs) (12-15). Bone marrowderived stromal cells (BMSCs), from which OBs differentiate, possess the properties and functions of niche cells: namely CXC chemokine ligand 12 (CXCL12)-abundant reticular cells (CAR cells) (16) and Nestin ${ }^{+}$MSCs. It has been reported that purified HSCs specifically home to Nestin ${ }^{+}$MSCs in the bone marrow of irradiated mice and $\mathrm{Nestin}^{+}$cell depletion results in a significantly compromised homing process (17). BMSCs have been shown to regulate the proliferation of HSCs rather than quiescence and support HSC maintenance and engraftment (17). Furthermore, BMSCs are characterized by their multi-differentiation potential. Our previous study demonstrated that OBs differentiated from BMSCs supported the maintenance and multipotency of HSPCs from umbilical cord blood in a 2D-culture system (18), suggesting that MSCs and OBs are suitable candidates with wich to build a novel HSP culture system.

In vitro $\mathrm{HSC}$ research is commonly carried out by culturing cells as monolayers using conventional tissue culture techniques. Although the contribution of conventional hematopoietic culture systems to the knowledge of human HSC biology is unquestionable, they lacked the three-dimensional (3D) architecture, thus failing to mimic the in vivo HSC niche, described as a three-dimensional microenvironment within the subendosteal region of bone marrow (1-3). In recent years, in vitro 3D-cultures of HSPCs have been shown to obviously be superior to bi-dimensional (2D) culture, which consists of HSPCs plus either sole MSCs or OBs, or both $(10,19,20)$. These findings imply that a 3D architecture is important to mimic physiological conditions ex vivo.

Bone marrow is located in both long bones (e.g., femur) and flat bones (e.g., calvaria); however, bio-derived bone scaffolds (BDBS) which are made from human femurs can preserve the natural spongy architecture of trabecular bones and more closely mimic the HSC niche in vivo. In our previous study, we utilized OBs and BDBS to create a 3D culture system, which was primarily demonstrated to support the maintenance and expansion of HSPCs in vitro, and this system was obviously superior to $2 \mathrm{D}$ culture systems (21). Taking into consideration the deep understanding of the HSC niche, in the present study, we used a mixture of MSCs and OBs differentiated from BMSCs and BDBS to improve our 3D-Mix culture system and to explore their synergized function on the BDBS, illustrating that its features can more closely mimic those of the HSC niche. Our data demonstrate that the 3D-Mix culture system has some features more similar to those of the HSC niche in supporting the maintenance and expansion of HSPCs in vitro.

\section{Materials and methods}

Preparation of BDBS. The BDBS characterized with respect to natural porosity, pore size and minerals were made from the human skeleton and were manufactured by the Division of Stem Cell and Tissue Engineering (Laboratory of Biotecherapy, Sichuan University, Chengdu, China). The preparation of BDBS involved a process through which a series of physical and chemical procedures were performed to drastically wipe off the main antigens, such as cells and lipoproteins, apart from bone morphogenetic protein, collagen and salinity (22), which included degreasing, partial deproteinization, decalcification and extensive washing with distilled water. Finally, the BDBS were lyophilized and sterilized by ${ }^{60} \mathrm{Co}$ gamma-ray irradiation $\left(20-25 \times 10^{3} \mathrm{~Gy}\right)$ before being stored at $4^{\circ} \mathrm{C}$. The human BDBS were cut into sections $(1.0 \times 0.5 \times 0.5 \mathrm{~cm})$ in order to fit into wells of 24-well plates, and soaked in neonatal bovine serum for $6 \mathrm{~h}$. They were then soaked in Dulbecco's modified Eagle's medium (DMEM) with $10 \%$ neonatal bovine serum again for $12 \mathrm{~h}$ before being used. The morphology of the BDBS was characterized by scanning electron microscopy.

Isolation and culture of human BMSCs. Heparinized human bone marrow cells were obtained from the posterior iliac crest of healthy volunteers with informed consent in accordance with the Declaration of Helsinki and following the approval of the Institutional Review Board of Sichuan Provincial Hospital. Bone marrow mononuclear cells (MNCs) were isolated after Ficoll-Hypaque (Sigma Diagnostics, St. Louis, MO, USA) gradient centrifugation at $400 \mathrm{x} \mathrm{g}$ for $30 \mathrm{~min}$ and plated in $25 \mathrm{~cm}^{2}$ cell culture flasks with expansion medium containing L-DMEM (Gibco Life Technologies, Paisley, UK), $10 \%$ fetal bovine serum (FBS; Gibco-BRL, Grand Island, NY, USA), $100 \mathrm{U} / \mathrm{ml}$ penicillin, $100 \mu \mathrm{g} / \mathrm{ml}$ streptomycin, $0.29 \mathrm{mg} / \mathrm{ml} \mathrm{L-glutamine} \mathrm{and} 3 \mathrm{mg} / \mathrm{ml} \mathrm{HEPES}$ buffer (R\&D Systems, Minneapolis, MN, USA) at $37^{\circ} \mathrm{C}$. After $72 \mathrm{~h}$, the non-adherent cells were discarded, and half medium change was performed twice a week. When the adherent MSCs grew to $>80 \%$ confluence, they were detached with $0.05 \%$ trypsin/0.53 mmol/1 EDTA (Sigma Diagnostics), and inoculated at $5 \times 10^{3}$ cells $/ \mathrm{cm}^{2}$ in 3 or 4 fresh tissue culture flasks.

Induction of differentiation of human BMSCs into OBs. When the MSCs at passage 3 achieved close to $60 \%$ confluence, the L-DMEM medium was discarded and replaced with osteogenic medium (F12 medium containing $10 \% \mathrm{FBS}, 10^{-8} \mathrm{~mol} / 1$ dexamethasone, $50 \mu \mathrm{g} / \mathrm{ml}$ ascorbic acid and $10 \mathrm{mmol} / 1$ $\beta$-sodium glycerol phosphate) to induce MSC differentiation into OBs. After 10 days of half changing the medium daily, the F12 medium was removed and was replaced with L-DMEM medium again. The osteogenic differentiation of the MSCs was assessed by alkaline phosphatase (ALP) staining, Alizarin Red S staining and by the analyses of the expression of type I collagen and osteocalcin.

ALP staining. ALP staining was performed using the Gomori modified calcium-cobalt method, as previously described (23). 
Briefly, the cells were fixed with a solution of $95 \%$ alcohol for $10 \mathrm{~min}$, and then incubated with ALP at $37^{\circ} \mathrm{C}$ for 4 to $6 \mathrm{~h}$. Subsequently, the cells were stained with solutions of $2 \%$ cobalt nitrate and $1 \%$ ammonium sulfide. After being air-dried, the cells on slides were mounted and observed under a light microscope (Olympus BX51, Olympus, Tokyo, Japan). Cells that were positive for ALP were stained a brown or tan color.

Alizarin Red S staining. Alizarin Red S staining was used to detect any calcified nodules in the cells, as previously described (24). The cells were fixed with $90 \%$ ethanol at room temperature for $1 \mathrm{~h}$ and stained with $40 \mathrm{mmol} / \mathrm{l}$ Alizarin Red S (Sigma) ( $\mathrm{pH} 4.2$ ) for $30 \mathrm{~min}$. The mineralized nodules were observed under a light microscope (Olympus BX51, Olympus).

Immunohistochemistry. The expression levels of type I collagen and osteocalcin were detected by immunohistochemistry. The cells on slides were fixed with cold acetone for $30 \mathrm{~min}$ at room temperature and incubated with blocking solution for $30 \mathrm{~min}$ at $37^{\circ} \mathrm{C}$. Subsequently, the slides were incubated with primary antibodies specific for type I collagen (Cat. no. MAB8887, Millipore, Temecula, CA, USA) and osteocalcin (cat. no. sc-74495, Santa Cruz Biotechnology, Inc., Santa Cruz, CA, USA) overnight at $4^{\circ} \mathrm{C}$. The following day, the cells were rinsed with PBS and incubated at $37^{\circ} \mathrm{C}$ for $40 \mathrm{~min}$ in the dark with fluorescein isothiocyanate (FITC, green)- or rhodamine (red)-conjugated secondary antibodies (Molecular Probes, Carlsbad, CA, USA). Furthermore, the nuclei were stained with $5 \mathrm{mg} / \mathrm{ml}$ DAPI (blue). The stained slides were observed under a laser confocal microscope (Olympus Fluoview FV500, Olympus).

Establishment of ex vivo hematopoietic culture systems (3D and $2 \mathrm{D})$. The 3D-culture system was established as follows: i) The OBs differentiated from the BMSCs for 10 days and the BMSCs at passage 3 were harvested and mixed at a ratio of 1:1 to act as seed cells. ii) Bio-derived bone blocks were soaked in L-DMEM for 2 days and then in FBS for $2 \mathrm{~h}$ before seeding the cells to facilitate cell adherence. iii) The mixture of BMSCs and OBs (1:1) was suspended into $8 \times 10^{6}$ cells $/ \mathrm{ml}$ in $20 \mu \mathrm{l}$ fresh L-DMEM and carefully dripped into prepared bone blocks to avoid overflow followed by being incubation at $37^{\circ} \mathrm{C}$ with $5 \% \mathrm{CO}_{2}$ in an incubator for $4 \mathrm{~h}$ to allow cell adherence. The bone blocks were then completely immersed in the L-DMEM medium supplemented with $10 \%$ FBS, $100 \mathrm{U} / \mathrm{ml}$ penicillin, $100 \mu \mathrm{g} / \mathrm{ml}$ streptomycin, $0.29 \mathrm{mg} / \mathrm{ml} \mathrm{L}$-glutamine and $3 \mathrm{mg} / \mathrm{ml}$ HEPES buffer. They were then grown to confluence for 7-10 days and treated with ${ }^{60} \mathrm{Co}$ gamma irradiation for $10 \mathrm{~min}$ (25 Gy). A 3D-culture system with only MSCs or OBs differentiated from BMSCs as seed cells was designed as the experimental control group, respectively. In addition, an irradiated (25 Gy) mixture of cells $\left(5 \times 10^{4}\right.$ cells $)$ was seeded and cultured in a 24-well plate to build the 2D system. In total, we used 4 culture systems (2D-Mix, 3D-OB, 3D-MSC and 3D-Mix).

Cultivation of purified $C D 34^{+}$cells from human umbilical cord blood $(U C B)$ in the $3 D$ or $2 D$ culture system. Human UCB cells were obtained from mothers at the end of full-term deliveries at Sichuan Provincial People's Hospital after obtaining written informed consent and the approval of the Institutional Review
Board of Sichuan Provincial Hospital. The UCB samples was incubated with $0.5 \%$ (w/v) methyl cellulose dissolved in Hanks' solution at room temperature for $30 \mathrm{~min}$ to sedimentate the UCB red blood cells before the MNCs were collected by FicollPaque centrifugation and the $\mathrm{CD} 34^{+}$cells were then enriched from the MNCs by using immune-magnetic beads (EasySep CD34 positive selection kit) and suspended in $20 \mu \mathrm{l}$ MyeloCult H5100 medium (both from StemCell Technologies Inc., Vancouver, BC, Canada) followed by being seeded into the 3D or 2D culture system and cultured with Myelo-Cult medium containing $1 \mu \mathrm{M}$ freshly dissolved hydrocortisone without exogenous cytokines. After 2 weeks of co-culture, the bone blocks were vigorously washed 3 times with phosphate-buffered saline (PBS) to collect the non-adherent cells, and then enzymatically dissociated with $0.125 \%$ trypsin $(0.78 \mathrm{mmol} / 1$ EDTA) followed by being pipetted and centrifuged at $300 \mathrm{x} \mathrm{g}$ for $15 \mathrm{~min}$ to harvest the adherent cells. Cells in the 2D culture system were also collected by trypsin digestion and centrifugation after 2 weeks of co-culture. The CD $34^{+}$cells from each culture system were subjected to various phenotypic and functional assays as described below.

Hoechst 33342/propidium iodide (PI) fluorescent staining. After 2 weeks of HSPC culture, the CD34+ cells were washed off and the bone blocks were transferred to a 24-well culture plate followed by the addition of Hoechst $(10 \mu \mathrm{g} / \mathrm{ml})$. The 24-well culture plate was incubated at $37^{\circ} \mathrm{C}$ for $10 \mathrm{~min}$ and then PI $(10 \mu \mathrm{l} / \mathrm{ml})$ was added. After $20 \mathrm{~min}$ of incubation at $4^{\circ} \mathrm{C}$, the 24 -well plate was taken out of the freezer before the bone blocks were observed under a fluorescence microscope (Olympus IX71, Olympus) to identify the viable/dead cells attached on the trabecular bone. The cells in the 2D culture system were also stained and observed under a fluorescence microscope as a control.

Quantitative PCR ( $q P C R)$. qPCR was performed for the detection of the mRNA expression of cyclin-dependent kinase inhibitor 1A (CDKN1A,p21) using DNA-binding SYBR-Green dye (Applied Biosystems, Foster City, CA, USA) for the detection of the PCR products. The primers used were as follows: p21 forward, 5'-GGAAGACCATGTGGACCTGT-3' and reverse, 5'-GGCGTTTGGAGTGG TAGAAA-3'; $\beta$-actin (internal reference) forward, 5'-GCAAGCAGGAGTATG ACGAG-3' andreverse,5'-CAAATAAAGCCATGCCAATC-3', which were purchased from Applied Biosystems. The cycling conditions were as follows: an initial denaturation at $95^{\circ} \mathrm{C}$ for $15 \mathrm{~min}$, followed by 50 cycles of denaturation at $94^{\circ} \mathrm{C}$ for $15 \mathrm{sec}$, annealing at $55^{\circ} \mathrm{C}$ for $15 \mathrm{sec}$, and extension at $72^{\circ} \mathrm{C}$ for $15 \mathrm{sec}$. The $\beta$-actin gene was used as a reference. The assay was replicated in 3 independent experiments.

Flow cytometry (FCM). To assess the percentage of HSPCs remaining in the $2 \mathrm{D}$ or $3 \mathrm{D}$ culture system before and after each culture, FCM was performed to analyze the immunophenotypes of CD34 and CD38. Briefly, a total of $5 \times 10^{5}$ test cells was suspended in $50 \mu \mathrm{l}$ PBS and stained with $10 \mu \mathrm{g} / \mathrm{ml}$ of purified mouse anti-human CD34/CD38 antibody (FcR) (Cat. no. 550760; Becton-Dickinson, Mountain View, CA, USA) at $4^{\circ} \mathrm{C}$ for $30 \mathrm{~min}$. The replicate sample incubated with isotype-matched antibodies was used as a control. Dead cells 
were eliminated by staining with 7AAD. After the cells were washed twice in the same buffer, a minimum of $2 \times 10^{4}$ events of each sample was analyzed on a FACS cell sorter using Cell Quest software (Becton-Dickinson).

Colony-forming unit (CFU) assay and morphological examination. The colony-forming assay was performed to assess the colony-forming ability of the cells. Briefly, the CD34+ cells from the initial UCB samples or the cultured cells were incubated in methylcellulose medium with recombinant cytokines (MethoCult GF+ H4435; StemCell Technologies Inc.) at $2 \times 10^{3}$ in $35-\mathrm{mm}$ tissue culture dishes (Costar, Lowell, MA, USA). The dishes were incubated at $37^{\circ} \mathrm{C}$ in a humidified atmosphere with $50 \mathrm{ml} / 1 \mathrm{CO}_{2}$ in air. All cultures were carried out in triplicate. After 14 days of culture, colonies belonging to burst-forming unit-erythroid (BFU-E), colony-forming unit-granulocyte/macrophage (CFU-GM), colony-forming unit-macrophage (CFU-M) and colony forming unit-granulocyte/erythroid/macrophage/megakaryocyte (CFU-GEMM) consisting of $\geq 50$ cells were scored under an inverted microscope (Olympus IX81, Olympus). To assess the accuracy of in situ identification, individual colonies were selected and stained with Wright's staining (Merck, Darmstadt, Germany) for the morphological identification of cells.

Long-term culture initiating cell (LTC-IC) assay. A modified LTC-IC assay was performed as previously described (25). Briefly, irradiated (80 Gy) mouse bone marrow stromal cells (M2-10B4, American Type Culture Collection, Rockville, MD, USA) were seeded at $10^{5}$ cells/well in 96-well flat-bottomed plates as a feeder layer. $\mathrm{CD} 34^{+}$cell subpopulations purified from UCB or those isolated from cultured cells by sorting with a FACSVantage (Becton-Dickinson) were seeded at a limiting dilution on the feeder layer in serum-containing medium. For each evaluation, at least 3 cell concentrations were used with 24 replicates per concentration. The plates were incubated at $37^{\circ} \mathrm{C}, 5 \% \mathrm{CO}_{2}$ with weekly half medium exchanges. After 5 weeks of culture, the cells were harvested and transferred to methylcellulose medium with recombinant cytokines (MethoCultGFt H4434V; StemCell Technologies Inc.) for colony-forming assays. After 14 days of culture, colonies with $>50$ cells were counted to assess LTC-IC activities and we calculate the frequency of LTC-IC according to the manufacturer's instructions (StemCell Technologies Inc.).

NOD/SCID repopulating cell (SRC) assay. Human cord blood CD34+ cells were cultured for 14 days in 4 culture systems (2D-Mix, 3D-OB, 3D-MSC and 3D-Mix). The cells were harvested, suspended in $1 \mathrm{ml} \alpha$-MEM and injected intravenously via the tail vein into 8-week-old, sub-lethallyirradiated (2.5 Gy) NOD/SCID mice ( $n=40$; Central Institute for Experimental Animals, Institutes for Biological Sciences, Shanghai, China). This animal experiment was approved by the Institutional Animal Care and Use Committee of the Sichuan Provincial People's Hospital. Each mouse received cells equivalent to $10^{4} \mathrm{CD} 34^{+}$cells together with irradiated (15 Gy using a ${ }^{60} \mathrm{Co} \gamma$-irradiator) non-repopulating CD34- cells as accessory cells. The recipients were sacrificed by carbon dioxide asphyxiation 8 weeks following transplantation, and bone marrow MNCs were harvested by Ficoll density gradient centrifugation and stained with FITC-labeled anti-human CD45 antibody to analyze for the presence of human $\mathrm{CD} 45^{+}$cells by FCM. Mice were considered positive for human HSC engraftment when at least $0.1 \% \mathrm{CD} 45^{+}$human cells were detected in the mouse bone marrow cells. The SRC frequency was calculated based on the Poission distribution using the equation $\mathrm{Pi}=\mathrm{e}(-\mathrm{N}) \times(\mathrm{Ni} / \mathrm{i} !)$ at $\mathrm{P} 0$, as previously described $(26,27)$. PCR analysis using human specific $17 \alpha$-satellite gene expression (17 $\alpha$-satellite, 5'-ACGGGATAACTGCACCTAAC-3'; 5'-CCATAGGAGGGTTCAACTCT-3') was performed to confirm the FCM results.

Enzyme-linked immunosorbent assay (ELISA). After 2 weeks of HSPC culture, the media from 4 culture systems were clarified at 3,500 rpm for $5 \mathrm{~min}$, and the supernatants were subjected to quantitative ELISA for the secreted fibronectin, collagen IV, vitronectin and laminin using commercially available fibronectin, collagen IV, vitronectin and laminin ELISA kits (RayBiotech Inc., Norcross, GA, USA) according to the manufacturer's instructions.

Western blot analysis. The MSCs and OBs in the 4 culture systems were harvested, washed twice in cold 1X PBS (Gibco, Invitrogen), and subsequently lysed in ice-cold RIPA lysis buffer [ $50 \mathrm{mmol} / \mathrm{l}$ Tris- $\mathrm{HCl}(\mathrm{pH} 8.0), 150 \mathrm{mmol} / \mathrm{l} \mathrm{NaCl}, 0.1 \%$ SDS, $1 \% \mathrm{NP}-40,0.25 \%$ sodium deoxycholate and $1 \mathrm{mmol} / \mathrm{l}$ EDTA] with freshly added protease inhibitor cocktail (Calbiochem, Shanghai, China). After $30 \mathrm{~min}$ of lysis on ice, the cell lysates were cleared by centrifugation at $12,000 \mathrm{x} \mathrm{g}$, and the protein concentration was measured using the BCA Protein assay kit (Pierce, Pittsburgh, PA, USA). Equal amounts of the protein samples were subjected to $10 \%$ sodium dodecyl sulfate-polyacrylamide gel electrophoresis (SDS/PAGE) and blotted onto PVDF membranes (GE Healthcare Biosciences, Piscataway, NJ, USA). The membranes were blocked with PBS-T containing 5\% non-fat dry milk and incubated with anti-angiopoietin-1 (cat. no. ab76956; Abcam, Cambridge, MA), anti-osteopontin (cat. no. sc-10591; Santa Cruz Biotechnology, Inc.), anti-stem cell factor (SCF; cat. no. ab52603), CXCL12 (cat. no. ab25117), RUNX2 (cat. no. ab76956) (all 3 from Abcam, Cambridge, MA, USA) and anti-glyceraldehyde 3-phosphate dehydrogenase (GAPDH) (cat. no. sc-5565; Santa Cruz Biotechnology, Inc.) antibodies. Membrane-bound first-step antibodies were reacted with horseradish peroxidase (HRP)-conjugated secondary antibody (Sigma). The bands were visualized with enhanced chemiluminescence (Millipore, Billerica, MA, USA). Protein bands were quantified using ImageJ software 1.43 (NIH, Bethesda, MD, USA) and normalized to the levels of GAPDH (loading control).

Statistical analysis. The data were presented as the means and standard error. Statistical comparisons were performed using the two-sided Student's t-test. Values of $\mathrm{p}<0.05$ and $\mathrm{p}<0.01$ were considered to indicate statistically significant differences.

\section{Results}

HSCs derived from $3 D$ culture system exhibit a morphological characterization similar to the HSC niche. It is now clear that MSCs and OBs are two crucial components of the HSC niche 

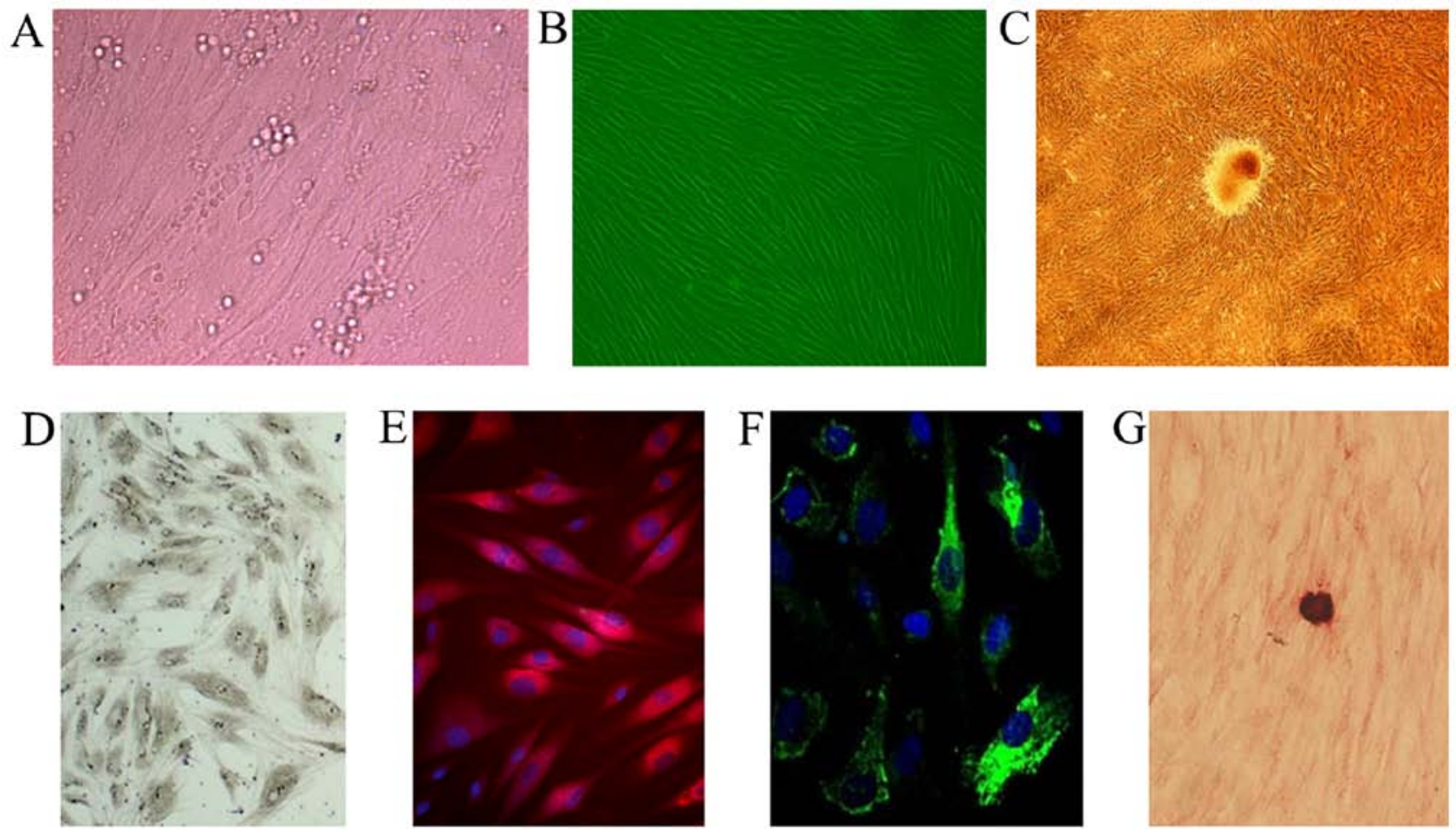

Figure 1. Osteoblasts differentiated from human marrow mesenchymal stromal cells (MSCs). (A) Morphology of HSPCs in the 2D system. The mixture of MSCs and osteoblasts (OBs) induced from the bone marrow-derived MSCs (BMSCs) as seed cells was seeded in a 24-well plate to build the 2D system in which the HSPCs were cultured for 2 weeks (x200 magnification). (B) Morphology of human MSCs of the first generation (x200 magnification); (C) morphology of osteoblasts differentiated from MSCs after 10 days (x40 magnification); (D) Gomori staining for alkaline phosphatase (ALP) expression in osteoblasts after 8 days (x200 magnification); (E) assay for osteocalcin expression with rhodamine-conjugated monoclonal antibody after 14 days (x400 magnification); (F) assay for collagen I by immunohistochemistry after 10 days (x100 magnification); (G) Alizarin S staining for mineralized nodule formation after 21 days (x100 magnification).

in adult bone marrow. Thus, we selected a mixture of MSCs and OB-like cells differentiated from MSCs to mimic the HSC niche and to build an HSC in vitro culture system in our study. Our primary MSCs were derived from the bone marrow of healthy adults, and were then differentiated into OB-like cells by culture in osteogenic medium for 10 days (Fig. 1B and C). We then performed morphological and histological analyses of the MSCs differentiated into OBs. Our results revealed that the OB-like cells differentiated from the MSCs were a heterogeneous population, as evidenced by asynchronous differentiation and the dynamic expression of osteogenic markers, including ALP, type I collagen, osteocalcin and calcium nodules (Fig. 1D-G).

Since the BDBS material was derived from human long bone (femurs) where adult hematopoietic tissues are mainly located, they thus supplied a spatial structure very similar to the HSC niche. Our study demonstrated an excellent biocompatibility of the bio-derived bone with human BMSCs and OBs differentiated from MSCs in vitro. After seeding the BMSCs and OBs differentiated from MSCs into the bio-derived bone for 7-10 days, the cells grew along with the inner surfaces of bone trabecular pores and formed a stable fibrous 3D structure. As shown by scanning electron and inverted microscopy, the cells tightly attached to the surface of the trabecular bone and secreted an amount of extracellular matrix (ECM) components which appeared elliptical with pseudopodal extensions and filled the intertrabecular cavity of cancellous bone (Fig. 2A-D). These features indicated a 'biomimetic HSC niche' with BDBS as a 3D scaffold, and a mixture of MSCs and OB-like cells as HSCs. Compared to the 3D culture system, the mixture of MSCs and OB-like cells in the 2D system was flat in shape and produced a lower amount of ECM components (Fig. 1A).

In order to determine whether the 3D-Mix system is able to supply a long-term culture of HSC in vitro, the frequency of live and dead feeder cells (MSCs and OB-like cells) on trabecular bone of BDBS after 2 weeks of HSPC culture was investigated by Hoechst 33342/PI fluorescent staining (Fig. 2E-F). It was shown that feeder cell death was $8.1 \%$ in the 3D-Mix culture system, whereas feeder cell death in the 2D-Mix culture system reached $17.3 \%$ after 2 weeks (data not shown).

3D-Mix of MSCs and OB differentiated from MSCs provide a microenvironment closely similar to the HSC niche. It is known that the bone marrow niche plays key roles in the self-renewal, survival, and maintenance of HSCs by expressing many crucial molecules, including chemokines, growth factors, cell-surface and adhesion molecules (28). Thus, in this study, we examined the protein expression of angiopoietin-1, RUNX2, CXCL12, osteopontin and SCF, which are genes that control HSC properties, in our 2D and 3D culture system. The results of western blot analysis revealed that all these genes were highly expressed in the 3D-Mix, despite the fact that the expression of individual genes was slightly lower than or close to that of the other culture systems, such as the expression of osteopontin in the 3D-OB (was higher), and that of CXCL12 in the 3D-MSC (was higher) systems. These findings implied that the interactions of human BMSCs and OBs in the 3D-Mix may contribute to a more comprehensive and balanced expression of cytokines, 

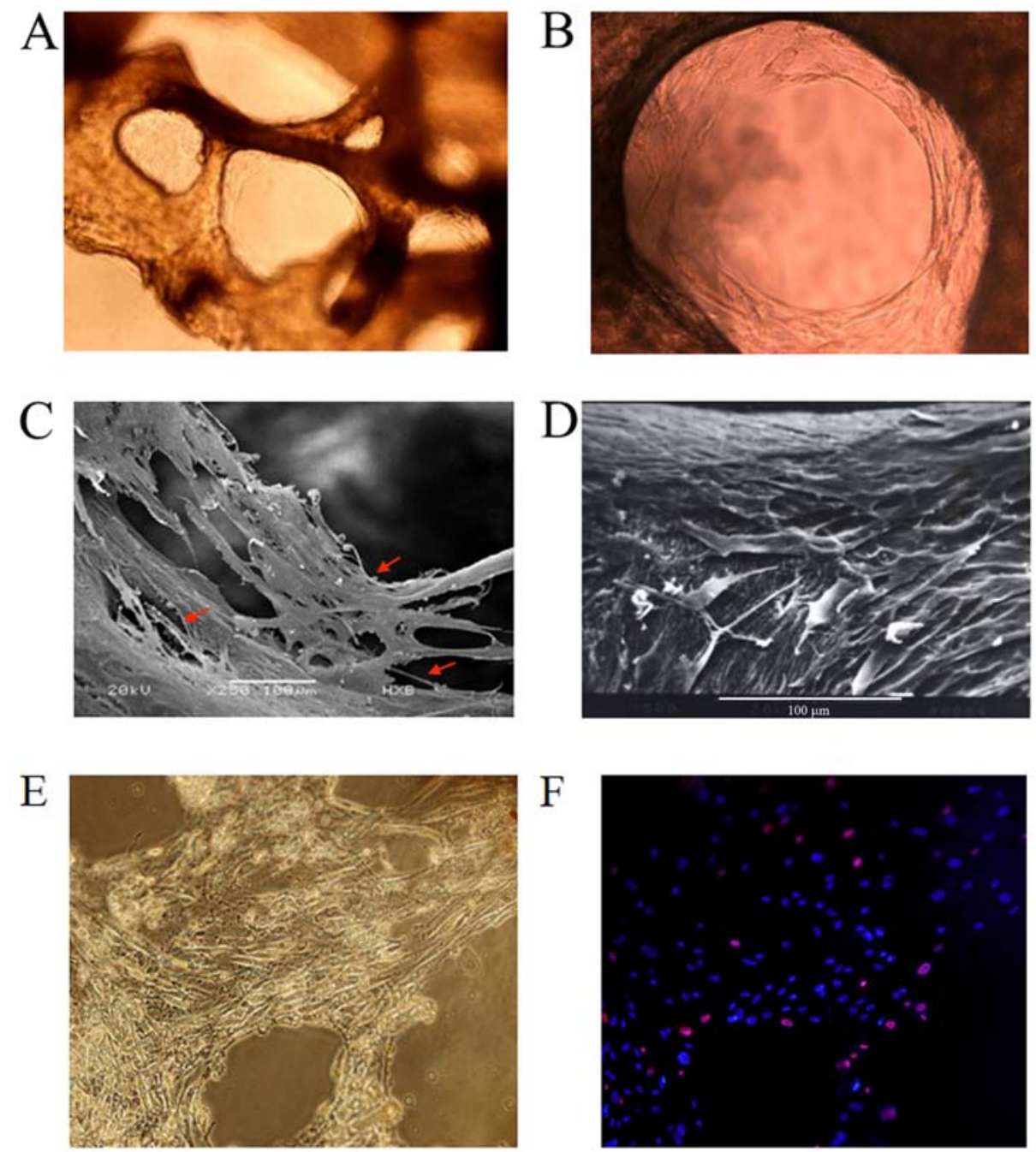

Figure 2. The cells grew after seeding human bone marrow stromal cells (BMSCs) and osteoblasts differentiated from mesenchymal stromal cells (MSCs) onto bio-derived bone for 7-10 days. (A and B) Human BMSCs and osteoblasts differentiated from MSCs on human bio-derived bone scaffolds examined under an inverted microscope after 10 days [(A) x100 magnification; (B) x200 magnification]; (C and D) human BMSCs and osteoblasts differentiated from MSCs on human bio-derived bone scaffolds examined under a scanning electron microscope after 10 days [(C and D) x1,000 magnification; arrows indicate ECM components]; (E and F) Hoechst 33342/propidium iodide (PI) fluorescent staining to examine the frequency of live and dead feeder cells on trabecular bone of bio-derived bone scaffolds after 2 weeks of HSPC culture. (E) Observation under an inverted microscope; (F) observation under a fluorescence microscope. Blue indicates the live cells and red indicates the dead cells.

which likely more closely resembles the physiological state of the HSC niche and is more beneficial to HSPC in vitro culture compared with the other culture systems (Fig. 3A).

The HSC microenvironment is enriched with ECM proteins, such as fibronectin, collagen IV, vitronectin and laminin, which are an essential part of the HSC niche (29). Thus, in this study, the culture systems were subjected to ELISA to determine the presence of these molecules. It was observed that all these ECM molecules were highly expressed at the protein level in the 3D-Mix, although the levels of individual ECM molecules, such as laminin and collagen IV were slightly lower than or similar to those in the 3D-MSC system (Fig. 3B-E). These findings indicated that the interactions of human BMSCs and OBs in the 3D-Mix provided most comprehensive ECM protein amounts to support and modulate HSPCs in vitro, compared to the other 3 systems.

Abundant growth of $\mathrm{CD} 34^{+}$cells in the $3 D$-Mix system. It is known that $\mathrm{CD} 34$ positivity is used as the most common immune phenotype for human HSPCs, and CD $34^{+} \mathrm{CD} 38^{-}$cells are regarded as the more primitive cell subpopulation. After 2 weeks of HSPC culture in the 3D or 2D system, the cells were harvested and subjected to FCM to count the number of $\mathrm{CD} 34^{+}$cells, $\mathrm{CD} 34^{+} \mathrm{CD} 38^{-}$cells representing long-term repopulating cells (LTRCs) and $\mathrm{CD} 34^{+} \mathrm{CD} 38^{+}$cells representing short-term repopulating cells (STRCs) in these systems (Fig. 4A). It was observed that the levels of expansion in the total $\mathrm{CD} 34^{+}$cell numbers in the 3D-Mix were significantly higher $(\mathrm{p}<0.05)$, compared to the other 3 systems (3D-MSC, $3 \mathrm{D}-\mathrm{OB}$ and 2D-Mix). The yield of $\mathrm{CD} 34^{+} \mathrm{CD} 38^{-}$cells in the $3 \mathrm{D}-\mathrm{Mix}$ was significantly higher than that in the $3 \mathrm{D}-\mathrm{OB}$ and 2D-Mix, although there was no statistically significant difference compared with the 3D-MSC system. Moreover, the expansion of $\mathrm{CD} 34^{+} \mathrm{CD} 38^{+}$cells in the 3D-Mix was also significantly improved when compared to that in the 3D-MSC and 2D-Mix ( $<<0.05)$, although the number of $\mathrm{CD} 34^{+} \mathrm{CD} 38^{+}$cells in the 3D-Mix was only slightly higher than that of the 3D-OB system (Table I). Overall, these findings indicated that the 
Table I. Umbilical cord blood HSC expansion over 7 days of culture.

\begin{tabular}{|c|c|c|c|c|}
\hline Group & 3D-Mix & 3D-MSC & 3D-OB & 2D-Mix \\
\hline $\mathrm{CD} 4^{+}$cells & $14.86 \pm 3.74$ & $7.69 \pm 1.67^{\mathrm{a}}$ & $11.52 \pm 4.58^{\mathrm{a}}$ & $2.63 \pm 1.17^{\mathrm{a}}$ \\
\hline $\mathrm{CD} 4^{+} \mathrm{CD} 38^{-}$cells & $5.42 \pm 1.07$ & $4.96 \pm 1.44$ & $1.52 \pm 0.53^{\mathrm{a}}$ & $0.57 \pm 0.16^{\mathrm{a}}$ \\
\hline $\mathrm{CD} 34^{+} \mathrm{CD} 38^{+}$cells & $12.65 \pm 4.42$ & $6.36 \pm 2.07^{\mathrm{a}}$ & $10.87 \pm 5.33$ & $2.09 \pm 1.35^{\mathrm{a}}$ \\
\hline
\end{tabular}

${ }^{\mathrm{a}} \mathrm{p}<0.05$ compared with 3D-Mix system. HSCs, hematopoietic stem cells.
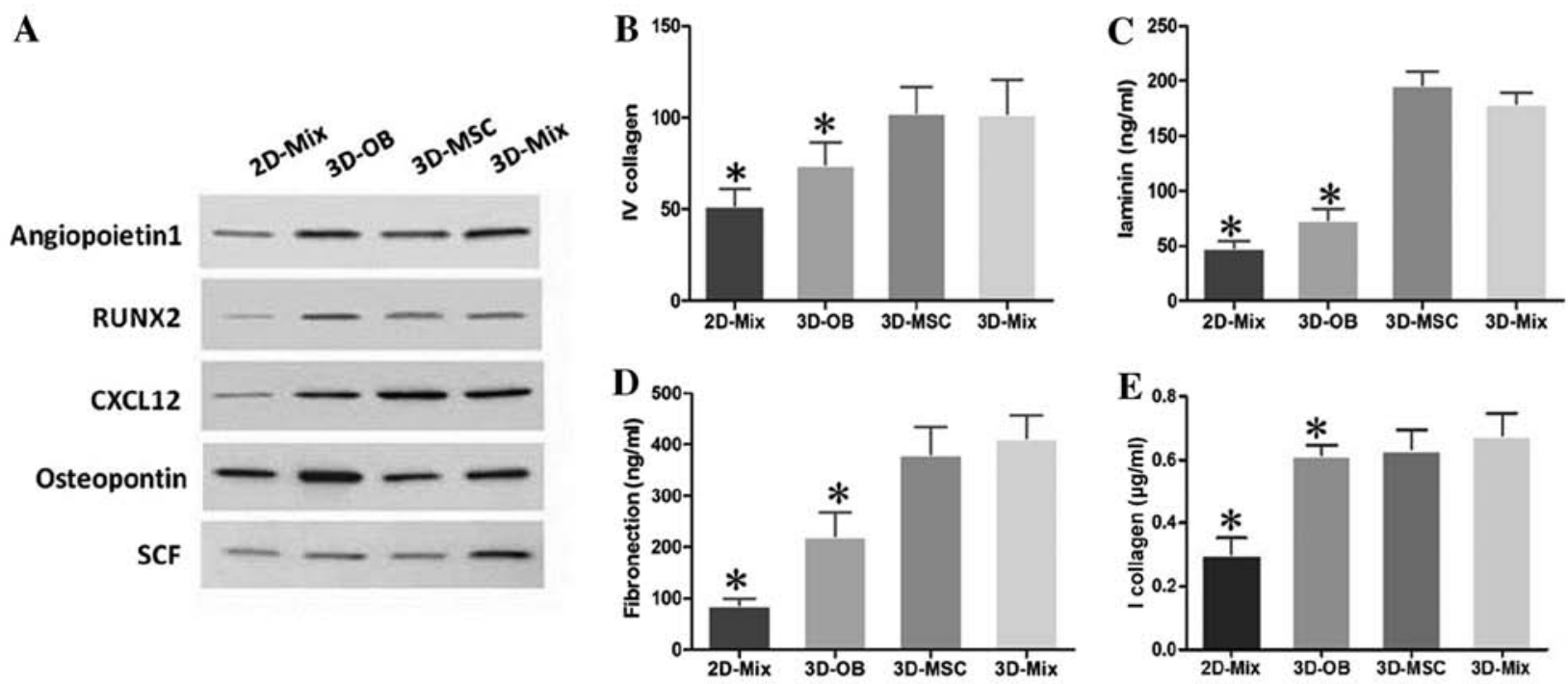

Figure 3. 3D-Mix of mesenchymal stromal cells (MSCs) and osteoblasts differentiated from MSCs supply a closely similar microenvironment. (A) Western blot analysis revealed the expression of angiopoietin-1, RUNX2, CXCL12, osteopontin and stem cell factor (SCF) in the 4 culture systems. (B-E) ELISA confirmed the expression of extracellular matrix proteins, such as fibronectin, collagen IV, vitronectin and laminin in the 4 culture systems. "p $<0.05$ compared with 3D-Mix system.

3D-Mix culture system had the advantages of both the 3D-OB and 3D-MSC systems, in that it did not only expand the total number of $\mathrm{CD} 34^{+}$haematopoietic cells, but also maintained these primitive cells in vitro.

A large proportion of HSCs is known to be in a quiescent state in the bone marrow niche (9). It was not apparent whether HSCs in this state enter the cell cycle at all. In this study, in order to address this issue, HSPCs from the culture systems were subjected to qPCR assay to determine the expression of $\mathrm{p} 21$, which is an essential regulator of the quiescence of HSCs (30). Our results revealed that p21 expression in the HSCs from the 3D-Mix system was significantly higher than that in the HSCs from the other culture systems, particularly in comparison with the $3 \mathrm{D}-\mathrm{OB}$ and $2 \mathrm{D}-\mathrm{Mix}$ systems, indicating that a large percentage of HSCs from the 3D-Mix was maintained in the G0 stage of the cell cycle. This suggested that the 3D-Mix possessed the ability to foster a large pool of quiescent HSCs, which is a critical niche characteristic in vivo (Fig. 4B).

3D-Mix system provides superior support for active multilineage hematopoiesis. Cultured $\mathrm{CD} 34^{+}$cells from the systems were also subjected to in vitro CFU assays to evaluate the proliferation and differentiation potential of hematopoietic progenitor cells. The $\mathrm{CD} 34^{+}$cells were sorted after 2 weeks of culture to avoid contamination with other cells and we performed methylcellulose assay to determine their potential clonal growth as, CFU-GEMM (termed CFU-MIX), CFU-GM and BFU-E. Colony formation assays revealed a significantly higher output of progenitors from the 3D-Mix compared to the other 3 culture systems $(\mathrm{p}<0.001)$. The distribution of CFUs was analyzed using an inverted microscope to observe the colony morphology, showing a significantly higher number of BFU-E in the 3D-Mix compared to the other 3 culture systems. Moreover, the frequencies of CFU-GM and CFU-MIX in the 3D-Mix were similar to those in the 3D-MSC and 3D-OB systems, respectively (Fig. 4C).

In parallel to the experiment on committed progenitors, we also examined the effects of the 3D-Mix on more primitive progenitors, as measured using the in vitro LTC-IC assay. $\mathrm{CD} 34^{+}$cells generated in the 4 culture systems were subjected to in vitro LTC-IC assay to determine whether they could preserve the ability to sustain long-term hematopoiesis. Following the initial 14-day culture period in the 4 culture systems, the output of $\mathrm{CD}^{+} 4^{+}$cells was plated on M2-10B4 for 5 weeks and then cultured in methylcellulose to analyze the LTC-IC-derived colony-forming cells. It was observed that the LTC-IC frequency in the 3D-Mix system was higher than that of the other 3 systems (Fig. 4D).

Human cord blood CD $34^{+}$cells were cultured for 14 days in the 4 culture systems. The cells were harvested, washed and injected into sublethally irradiated (2.5 Gy) NOD/SCID mice; 
$\mathbf{A}$

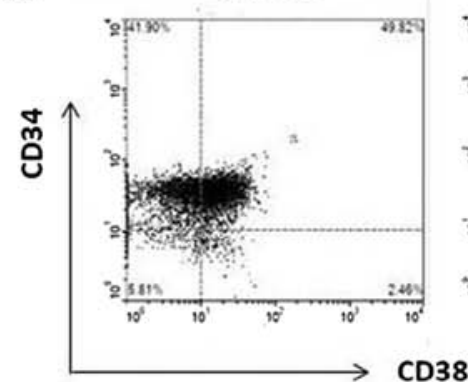

3D-MSC

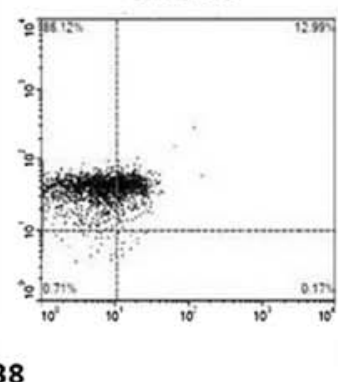

3D-OB

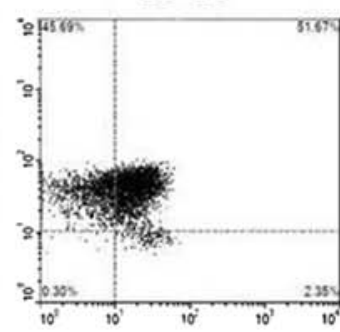

2D-Mix

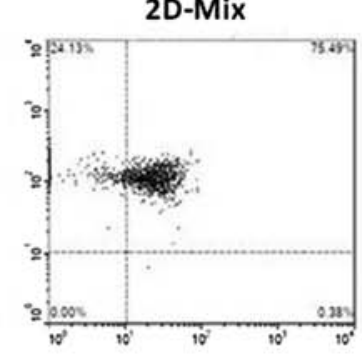

B

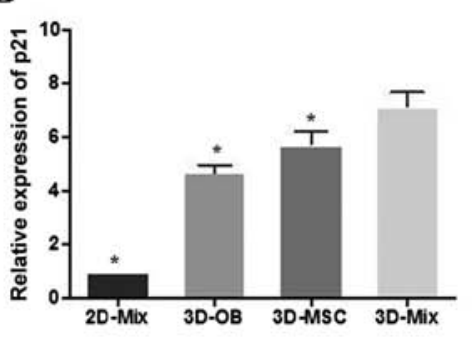

C

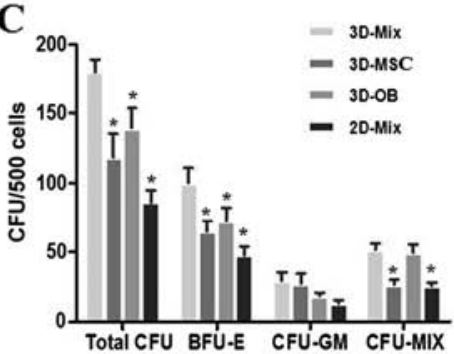

D

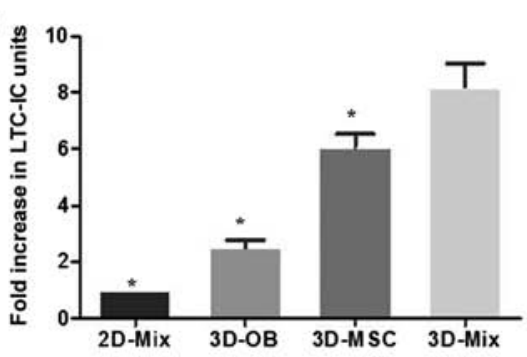

Figure 4.CD $34^{+}$cells in the 4 culture systems. (A) Flow cytometric analysis was used to count the number of CD $34^{+}$cells, $\mathrm{CD} 34^{+} \mathrm{CD} 38^{-}$cells and CD34 ${ }^{+} \mathrm{CD} 38^{+}$cells at 14 days; (B) qPCR assay was used to determine the expression of p21; (C) colony formation assays; (D) CD34 ${ }^{+}$cells generated in the 4 culture systems were subjected to in vitro long term culture initiating cell (LTC-IC) assays to determine whether they could preserve the ability to sustain long-term hematopoiesis. ${ }^{*} \mathrm{p}<0.05$ compared with 3D-Mix system.
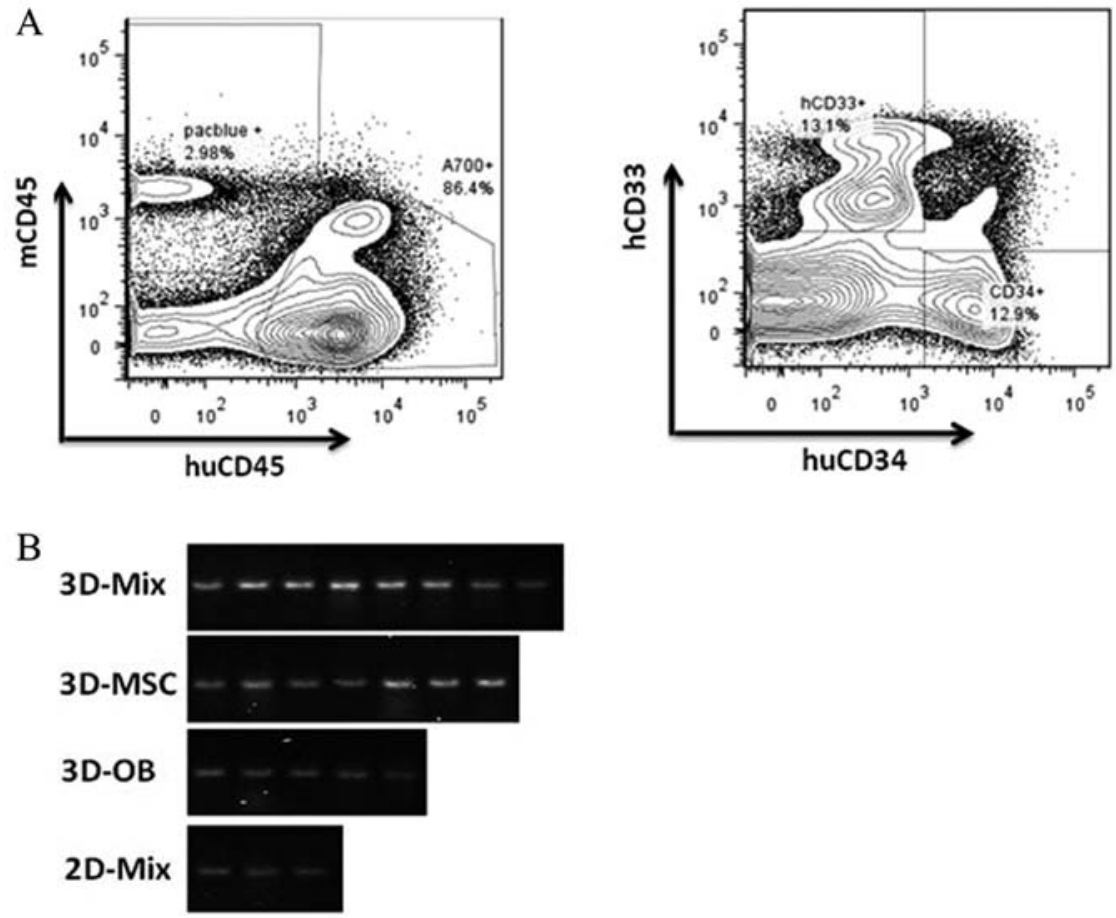

Figure 5. Determination of human hematopoietic reconstitution in NOD/SCID mice 8 weeks post-transplantation. (A) Transplantated bone marrow cells belonging to the 3D-Mix system from the mice were subjected to flow cytometric analysis; (B) PCR analysis of 17 $\alpha$-satellite gene expression demonstrated the presence of human hematopoietic cells in the bone marrow of NOD/SCID mice.

each mouse received cells equivalent to $10^{4}$ starting $\mathrm{CD} 34^{+}$cells. At 8 weeks post-cell transplantation, bone marrow cells were harvested from the recipients and subjected to FCM with an anti-human CD45 antibody. Mice were considered positive for human HSC engraftment when at least $0.1 \% \mathrm{CD} 45^{+}$human cells were detected in the mouse bone marrow cells. Bone marrow cells from positive mice were further analyzed by FCM using antibodies for human CD33 and CD34 antigens (Fig. 5A). We calculated the SRC concentration using the Poisson probability at P0. In our experiments, the positivity of human HSC engraftment in the 3D-Mix system were found to be the highest out of the 4 culture systems and cord blood CD $34^{+}$cells cultured in 
Table II. Expansion of SRCs in the 3D-Mix culture system.

\begin{tabular}{lccc}
\hline $\begin{array}{l}\text { Culture } \\
\text { system }\end{array}$ & Positive/total & \% Positive & $\begin{array}{c}\text { SRC } \\
\text { frequency }\end{array}$ \\
\hline 3D-Mix & $8 / 10$ & 80 & 1 in 5832 \\
3D-MSC & $7 / 10$ & $70^{\mathrm{a}}$ & 1 in $7901^{\mathrm{a}}$ \\
3D-OB & $5 / 10$ & $50^{\mathrm{a}}$ & 1 in $10745^{\mathrm{a}}$ \\
2D-Mix & $3 / 10$ & $30^{\mathrm{a}}$ & 1 in $16227^{\mathrm{a}}$ \\
\hline
\end{tabular}

${ }^{\mathrm{a}} \mathrm{p}<0.05$ compared with 3D-Mix system. SRC, SCID repopulating cell.

the 3D-Mix system were observed to have significantly higher SRC frequencies than the cells cultured in the other 3 systems $(\mathrm{p}<0.01$; Table II). Furthermore, PCR analysis of $17 \alpha$-satellite gene expression revealed the presence of human hematopoietic cells in the bone marrow of the NOD/SCID mice (Fig. 5B).

\section{Discussion}

An ideal HSPC culture system should possess both the characteristics of the long-term maintenance of the HSC pool and the continuousy promoting effects on the proliferation of the hematopoietic progenitor pool. However, the method with which to achieve this apparently contradictory and dynamic process in vitro continues to be an intriguing issue in HSPC culture research (1). To date, there is still not a suitable HSPC culture system to provide a suitable platform with which to study cellular biology and to be used in clinical practice by helping to achieve steady-state hematopoiesis in vivo. Although cytokine-driven culture which is supplemented with cytokines, such as fetal liver tyrosine kinase-3 ligand (FLT3-L), SCF, interleukin-3 (IL-3) and thrombopoietin (TPO) has been demonstrated to effectively promote expansion, it is difficult to maintain long-term HSPC culture due to the rapid onset of differentiation and the rapid loss of multipotency in culture, which thus renders this system insufficient for the clinical applications of these cells. By contrast, HSCs efficiently self-renew in their natural microenvironment (known as the HSC niche) in the bone marrow (31). Recently, studies have indicated that the HSC niche is responsible for HSC fate and have clarified the important regulatory component of the HSC niche (32-34). Therefore, closely mimicking the bone marrow niche ex vivo is considered to be an effective means of developing novel culture strategies to specifically regulate the balance of HSPC selfrenewal and proliferation. Studies have proven that this method is very effective $(19,35,36)$. With the in-depth understanding of the complex HSC niche, we further improved the HSPC culture protocol based on our previous study, which was demonstrated to initially have both properties of maintaining a certain number of the stem cell pool and continuously promoting the proliferation of progenitors, compared with other traditional culture systems. The HSC niche is an in vivo regulatory microenvironment where HSCs reside and maintain their capability of self-renewal and multipotency, and is composed of all types of cellular and humoral factors. OBs and BMSCs have long been revealed to be the two major crucial components of the HSC niche in bone marrow to support the maintenance, proliferation and differentiation of HSCs. OBs are derived from the MSCs in bone marrow. Namely, when MSCs commit to osteogenesis, they differentiate into osteoprogenitors that progress into pre-osteoblasts, OBs, and finally into osteocytes that mineralize and form bone (37). Actually, the osteoblastic niche has been proven to include osteoprogenitor cells, quiescent bone lining cells and active OBs (12). Sacchetti et al demonstrated that human $\mathrm{CD} 45^{-} \mathrm{CD} 146^{+}$osteoprogenitor cells were able to transfer hematopoietic activity to an ectopic site (38). It was found that the deletion of OBs under the control of collagen a1 type I promoter led to a decrease in the numbers of HSCs (39). That was why we selected the OBs differentiated from human BMSCs to act as a candidate of feeder cells in this study, instead of directly using a mature OB cell line. Our experiments of the induction of the differentiation of human BMSCs to OBs indicated that the MSCs that differentiated into OBs were a heterogeneous population at various stages of differentiation, rather than just fully mature OBs. MSCs express high levels of HSC maintenance factor transcripts, including CXCL12, angiopoietin-1, SCF, IL-7 and osteopontin. Méndez-Ferrer et al identified a nestin-expressing MSC population (nestin ${ }^{+}$MSC) that is closely associated with putative HSCs. The depletion of nestin ${ }^{+}$MSCs by the inducible expression of diphtheriatoxin receptor in nestin-expressing cells caused the mobilization of $50 \%$ of HSCs to the spleen (40). In addition, in another study, the homing of transplanted progenitor cells into MSC-depleted recipients was shown to be reduced by $90 \%$ (41). Recently, Omatsu et al found that CAR cells are important in supporting the proliferation of HSPCs. Further data confirmed that CAR cells which express osteogenic and adipogenic genes are a form of osteogenic-adipo progenitor derived from MSCs (42). Therefore, in this study, we selected chose human BMSCs as the other candidate of feeder cells in our culture system. In order to more closely mimic a multi-cell state in the HSC niche to produce synergistic control of the balance between quiescence, self-renewal and proliferation in the HSPC culture system, we co-cultured BMSCs and OBs differentiated from MSCs without any cytokines in this study to create a novel culture system to support the maintenance and proliferation of HSPCs in vitro.

In order to mimic the physical conditions of the HSC niche, many groups have tested different materials as scaffolds to build a 3D culture system, which include natural materials, such as cellulose porous microspheres and collagen carriers, or synthetic materials, such as macroporous PEG hydrogels, porous biomatrix, porous polyvinyl formal resin, polyethylene terephthalate, colloidal crystals and porous gelatin microspheres $(13-15,43,44)$. In this study, we adopted bio-derived bone made from human femurs as a system scaffold which preserves the natural spongy architecture of trabecular bone to more closely mimic the HSC niche in vivo. This study demonstrated that the BDBS had good biocompatibility and more importantly, partially reserved calcium, phosphonium and other ECM proteins, which have been proven to promote the osteogenic differentiation of MSCs in vitro. It is known that $3 \mathrm{D}$ cultures allow the reconstruction of the complex tissue architecture, thus providing a better platform with which to study cellular biology. There is evidence to indicate that $3 \mathrm{D}$ culture systems are superior to traditional 2D systems as regards the maintenance and expansion of HSPCs, which 
proves that the spatial architecture is one of the most important physiological conditions in the stem cell niche and influences the biological behavior of HSCs $(37,45-47)$. The natural scale and pore size of bio-derived bone as a scaffold are close to the natural architecture of the HSC niche in vivo. In this study, the mixture of human BMSCs and OBs differentiated from MSCs attached to this natural scaffold very well (70-80\% attachment) (data not shown). These two cell populations were observed to grow in the porous network of trabecular bone and form a meshwork-like structure, while the HSCs embedded and grew in the intercellular spaces. 3D-MSC and 3D-OB systems are used as controls apart from the traditional 2D culture system $(10,19,20)$. We carried out extensive analyses of specialized microenvironments that were recognized by hematopoietic $\mathrm{CD}^{+}{ }^{+}$cells in the $3 \mathrm{D}$ culture system. The HSC niche is known to express many crucial molecules, which include chemokines, growth factors, cell-surface and adhesion molecules, and plays key roles in HSC self-renewal, survival and maintenance (28). Furthermore, the majority of the secreted factors which have been proposed to control HSC fate, such as angiopoietin-1, RUNX2, CXCL12, osteopontin and SCF are supplied by MSCs and OBs in the HSC niche (48). We found that the interactions of human BMSCs and OBs in the 3D-Mix system may contribute to a more comprehensive and balanced expression of cytokines, which more closely mimics the physiological state of the HSC niche and is more beneficial to HSPC culture in vitro compared with the other culture systems. The HSC microenvironment is enriched with ECM proteins, such as fibronectin, collagen IV, vitronectin and laminin, which are an essential part of the HSC niche (29). Thus, in this study, the culture systems were subjected to ELISA to determine the presence of these molecules. High levels of ECM molecules were found to be secreted by the MSCs and OBs differentiated from MSCs and deposited on the surface of the two cell populations, which means that they themselves were capable of adding a physiologically relevant dimension to the culture system. In addition, our data indicated that the interactions of human BMSCs and OBs in the 3D-Mix system provided a comprehensive amount of ECM proteins to support and modulate HSPC culture in vitro.

In the present study, phenotypic analysis of expanded cells by $\mathrm{FCM}$ revealed that the proportion of $\mathrm{CD} 34^{+} \mathrm{CD} 38^{-}$cells representing LTRC in the 3D-Mix system was significantly higher than that in the 3D-OB system, although there was no statistically significant difference in the yield of $\mathrm{CD} 34^{+} \mathrm{CD} 38^{+}$ cells (which represent STRCs) between them, which implies that MSCs play an important role in maintaining the quiescence HSCs in vitro. On the other hand, although half of the OBs in the 3D-OB system was replaced by human BMSCs in the $3 \mathrm{D}-\mathrm{Mix}$ system, the number of $\mathrm{CD} 34^{+} \mathrm{CD} 38^{+}$cells between the two culture systems exhibited no obvious difference, which implies that the interaction of MSCs with OBs also has an effect on promoting HPC proliferation in vitro. We also found that the yield of $\mathrm{CD} 34^{+} \mathrm{CD} 38^{+}$cells from the $3 \mathrm{D}$-Mix culture system was significantly higher than that from the 3D-MSC system, although the number of $\mathrm{CD} 34^{+} \mathrm{CD} 38^{-}$cells was slightly lower than that from the 3D-MSC system, indicating tht OBs differentiated from human BMSCs play a critical role in improving the proliferation of HSPCs in vitro. On the other hand, although half of the MSCs in the 3D-MSC system was replaced by OBs in the 3D-Mix system, the number of
CD $34{ }^{+} \mathrm{CD} 38^{-}$cells in the 3D-Mix was close to that in the 3D-MSC system, which implies that the interaction of OBs with MSCs also has the effect of promoting HSC self-renewal in vitro.

PCR analysis of $\mathrm{p} 21$, which is an essential regulator of the quiescence of HSCs, also confirmed the FCM results, which showed that p21 expression in the HSCs from the 3D-Mix system was significantly higher than that in the cells from the 3D-OB system and very close to that in the cells from the 3D-MSC system, indicating that a large percentage of HSCs from the 3D-Mix system was maintained in the G0 stage of the cell cycle. It is known that UCB LTC-ICs are present among the $\mathrm{CD} 4^{+} \mathrm{CD} 38^{-}$cell fraction and LTC-IC assays exhibit multilineage differentiation ability and major proliferative potential, which is regarded as a functional measure of selfrenewal (47). Our results demonstrated that the frequency of LTC-ICs from the 3D-Mix system was statistically higher than that of the other 3 culture systems, particularly the 3D-OB and 2D-Mix systems, which demonstrated that the 3D-Mix system contained a higher number of more primitive hematopoietic cells ex vivo compared with the other 3 systems, possessing the important ability of fostering a large pool of quiescent HSCs, which is a critical niche characteristic in vivo. In the SRC assay, which indicated the reconstituting ability of these cultured cells, the difference in the percentage of chimerism of human CD $45^{+}$cells among bone marrow cells of mice transplanted with cultured cells strongly suggested that the 3D-Mix more effectively supported ex vivo-generated HSCs with the ability to sustain and reconstitute long-term human hematopoiesis in vivo, compared with the other 3 systems.

In conclusion, our data demonstrate that the $3 \mathrm{D}$ culture system, established by the co-culture of BMSCs and OBs differentiated from MSCs on human BDBS, is a more comprehensive and balanced system, which not only continuously and effectively promotes the self-renewal and ex vivo expansion of HSPCs, but also maintains a large pool of primitive HPCs with superior phenotypic and functional attributes. Compared with the other 3 culture systems, the 3D-Mix culture system has some features which are more similar to those of the HSC niche, namely as regards the maintenance and expansion of HSPCs in vitro.

\section{References}

1. Vunjak-Novakovic G and Scadden DT: Biomimetic platforms for human stem cell research. Cell Stem Cell 8: 252-261, 2011.

2. Konopleva MY and Jordan CT: Leukemia stem cells and microenvironment: Biology and therapeutic targeting. J Clin Oncol 29: 591-599, 2011.

3. Konopleva M, Tabe Y, Zeng Z and Andreeff M: Therapeutic targeting of microenvironmental interactions in leukemia: Mechanisms and approaches. Drug Resist Updat 12: 103-113, 2009.

4. Sharma MB, Limaye LS and Kale VP: Mimicking the functional hematopoietic stem cell niche in vitro: recapitulation of marrow physiology by hydrogel-based three-dimensional cultures of mesenchymal stromal cells. Haematologica 97: 651-660, 2012.

5. Bird GA, Polsky A, Estes P, Hanlon T, Hamilton H, Morton JJ, Gutman J, Jimeno A, Turner BC and Refaeli Y: Expansion of human and murine hematopoietic stem and progenitor cells ex vivo without genetic modification using MYC and Bcl-2 fusion proteins. PLoS One 9: e105525, 2014.

6. Soufizomorrod M, Soleimani M, Hajifathali A, Mohammadi MM and Abroun S: Expansion of CD133 umbilical cord blood derived hematopoietic stem cells on a biocompatible microwells. Int J Hematol Oncol Stem Cell Res 7: 9, 2013. 
7. Bari S, Chu PP, Lim A, Fan X, Gay FP, Bunte RM, Lim TK, Li S, Chiu GN and Hwang WY: Protective role of functionalized single walled carbon nanotubes enhance ex vivo expansion of hematopoietic stem and progenitor cells in human umbilical cord blood. Nanomedicine (Lond) 9: 1304-1316, 2013.

8. Zhang J, Niu C, Ye L, Huang H, He X, Tong WG, Ross J, Haug J, Johnson T, Feng JQ, et al: Identification of the haematopoietic stem cell niche and control of the niche size. Nature 425: 836-841, 2003.

9. Arai F and Suda T: Maintenance of quiescent hematopoietic stem cells in the osteoblastic niche. Ann NY Acad Sci 1106: 41-53, 2007.

10. Di Maggio N, Piccinini E, Jaworski M, Trumpp A, Wendt DJ and Martin I: Toward modeling the bone marrow niche using scaffoldbased 3D culture systems. Biomaterials 32: 321-329, 2011.

11. Taichman RS, Reilly MJ and Emerson SG: Human osteoblasts support human hematopoietic progenitor cells in vitro bone marrow cultures. Blood 87: 518-524, 1996.

12. Wu JY, Scadden DT and Kronenberg HM: Role of the osteoblast lineage in the bone marrow hematopoietic niches. J Bone Miner Res 24: 759-764, 2009.

13. Bagley J, Rosenzweig M, Marks DF and Pykett MJ: Extended culture of multipotent hematopoietic progenitors without cytokine augmentation in a novel three-dimensional device. Exp Hematol 27: 496-504, 1999.

14. Li Y, Ma T, Kniss DA, Yang ST and Lasky LC: Human cord cell hematopoiesis in three-dimensional nonwoven fibrous matrices: In vitro simulation of the marrow microenvironment. J Hematother Stem Cell Res 10: 355-368, 2001.

15. Zhang Y, Chai C, Jiang XS, Teoh S-H and Leong KW: Co-culture of umbilical cord blood CD34p cells with human mesenchymal stem cells. Tissue Eng 12: 2161-2170, 2006.

16. Sugiyama T, Kohara H, Noda M and Nagasawa T: Maintenance of the hematopoietic stem cell pool by CXCL12-CXCR4 chemokine signaling in bone marrow stromal cell niches. Immunity 25 977-988, 2006

17. Méndez-Ferrer S, Michurina TV, Ferraro F, Mazloom AR, MacArthur BD, Lira SA, Scadden DT, Ma'ayan A, Enikolopov GN and Frenette PS: Mesenchymal and haematopoietic stem cells form a unique bone marrow niche. Nature 466: 829-834, 2010.

18. Huang X, Liu T, Meng W and Zhi W: Osteoblasts differentiated from human marrow bone mesenchymal stem cells support hematopoietic stem/progenitor cells from umbilical cord blood. Zhongguo Shi Yan Xue Ye Xue Za Zhi 14: 552-556, 2006 (In Chinese)

19. Raic A, Rödling L, Kalbacher $H$ and Lee-Thedieck $C$ : Biomimetic macroporous PEG hydrogels as 3D scaffolds for the multiplication of human hematopoietic stem and progenitor cells. Biomaterials 35: 929-940, 2014.

20. Miyoshi H, Ohshima N and Sato C: Three - dimensional culture of mouse bone marrow cells on stroma formed within a porous scaffold: Influence of scaffold shape and cryopreservation of the stromal layer on expansion of haematopoietic progenitor cells. J Tissue Eng Regen Med 7: 32-38, 2013.

21. Huang XB, Liu T, Meng WT, Deng L, Zhi Wa nd Zhu HL: Imitating human hematopoietic niche with osteoblasts derived from human marrow mesenchymal stem cells to sustain stem/progenitor cells proliferation. Chin J Hematol 27: 795-800, 2006 (In Chinese).

22. Xie H, Yang F, Deng L, Luo J, Qin T, Li X, Zhou GQ and Yang Z: The performance of a bone-derived scaffold material in the repair of critical bone defects in a rhesus monkey model. Biomaterials 28: 3314-3324,2007.

23. Sun F, Li X, Yang C, Lv P, Li G and Xu H: A role for PERK in the mechanism underlying fluoride-induced bone turnover Toxicology 325: 52-66, 2014

24. Huang YC, Zhu HM, Cai JQ, Huang YZ, Xu J, Zhou Y, Chen XH, Li XQ, Yang ZM and Deng L: Hypoxia inhibits the spontaneous calcification of bone marrow-derived mesenchymal stem cells. J Cell Biochem 113: 1407-1415, 2012.

25. Verfaillie CM: Direct contact between human primitive hematopoietic progenitors and bone marrow stroma is not required for long-term in vitro hematopoiesis. Blood 79: 2821-2826, 1992.

26. Chen J, Astle CM and Harrison DE: Development and aging of primitive hematopoietic stem cells in BALB/cBy mice. Exp Hematol 27: 928-935, 1999.

27. Chen J, Ellison FM, Eckhaus MA, Smith AL, Keyvanfar K, Calado RT and Young NS: Minor antigen h60-mediated aplastic anemia is ameliorated by immunosuppression and the infusion of regulatory T cells. J Immunol 178: 4159-4168, 2007.
28. Mendelson A and Frenette PS: Hematopoietic stem cell niche maintenance during homeostasis and regeneration. Nat Med 20: 833-846, 2014

29. Muth CA, Steinl C, Klein G and Lee-Thedieck C: Regulation of hematopoietic stem cell behavior by the nanostructured presentation of extracellular matrix components. PLoS One 8: e54778, 2013.

30. Cheng T, Rodrigues N, Shen H, Yang Y-g, Dombkowski D, Sykes M and Scadden DT: Hematopoietic stem cell quiescence maintained by p21cip1/waf1. Science 287: 1804-1808, 2000

31. Taichman RS: Blood and bone: Two tissues whose fates are intertwined to create the hematopoietic stem-cell niche. Blood 105 2631-2639, 2005

32. Catlin SN, Busque L, Gale RE, Guttorp P and Abkowitz JL: The replication rate of human hematopoietic stem cells in vivo. Blood 117: 4460-4466, 2011.

33. Kiel MJ, Yilmaz ÖH, Iwashita T, Yilmaz OH, Terhorst $\mathrm{C}$ and Morrison SJ: SLAM family receptors distinguish hematopoietic stem and progenitor cells and reveal endothelial niches for stem cells. Cell 121: 1109-1121, 2005.

34. Osawa M, Hanada K-i, Hamada $\mathrm{H}$ and Nakauchi H: Long-term lymphohematopoietic reconstitution by a single CD34-low/negative hematopoietic stem cell. Science 273: 242-245, 1996.

35. Raynaud CM, Butler JM, Halabi NM, Ahmad FS, Ahmed B, Rafii $S$ and Rafii A: Endothelial cells provide a niche for placental hematopoietic stem/progenitor cell expansion through broad transcriptomic modification. Stem Cell Res (Amst) 11: 1074-1090, 2013

36. de Barros AP, Takiya CM, Garzoni LR, Leal-Ferreira ML, Dutra HS, Chiarini LB, Meirelles MN, Borojevic R and Rossi MI: Osteoblasts and bone marrow mesenchymal stromal cells control hematopoietic stem cell migration and proliferation in 3D in vitro model. PLoS One 5: e9093, 2010.

37. Bianco P: Bone and the hematopoietic niche: A tale of two stem cells. Blood 117: 5281-5288, 2011.

38. Sacchetti B, Funari A, Michienzi S, Di Cesare S, Piersanti S, Saggio I, Tagliafico E, Ferrari S, Robey PG, Riminucci M and Bianco P: Self-renewing osteoprogenitors in bone marrow sinusoids can organize a hematopoietic microenvironment. Cell 131: 324-336, 2007.

39. Visnjic D, Kalajzic Z, Rowe DW, Katavic V, Lorenzo J and Aguila HL: Hematopoiesis is severely altered in mice with an induced osteoblast deficiency. Blood 103: 3258-3264, 2004.

40. Méndez-Ferrer S, Lucas D, Battista M and Frenette PS: Haematopoietic stem cell release is regulated by circadian oscillations. Nature 452: 442-447, 2008

41. Anthony BA and Link DC: Regulation of hematopoietic stem cells by bone marrow stromal cells. Trends Immunol 35: 32-37, 2014.

42. Omatsu Y, Sugiyama T, Kohara H, Kondoh G, Fujii N, Kohno K and Nagasawa T: The essential functions of adipo-osteogenic progenitors as the hematopoietic stem and progenitor cell niche. Immunity 33: 387-399, 2010.

43. Tun T, Miyoshi H, Aung T, Takahashi S, Shimizu R, Kuroha T, Yamamoto M and Ohshima N: Effect of growth factors on ex vivo bone marrow cell expansion using three - dimensional matrix support. Artif Organs 26: 333-339, 2002.

44. Nichols JE, Cortiella J, Lee J, Niles JA, Cuddihy M, Wang S, Bielitzki J, Cantu A, Mlcak R, Valdivia E, et al: In vitro analog of human bone marrow from 3D scaffolds with biomimetic inverted colloidal crystal geometry. Biomaterials 30: 1071-1079, 2009.

45. Chitteti BR, Bethel M, Voytik-Harbin SL, Kacena MA and Srour EF: In vitro construction of 2D and 3D simulations of the murine hematopoietic niche. In: Stem Cell Niche. Springer, pp43-56, 2013.

46. Hirabayashi Y, Hatta Y, Takeuchi J, Tsuboi I, Harada T, Ono K, Glomm WR, Yasuda M and Aizawa S: Novel three-dimensional long-term bone marrow culture system using polymer particles with grafted epoxy-polymer-chains supports the proliferation and differentiation of hematopoietic stem cells. Exp Biol Med 236 1342-1350, 2011

47. Tang Y, Chen J and Young NS: Expansion of haematopoietic stem cells from normal donors and bone marrow failure patients by recombinant hoxb4. Br J Haematol 144: 603-612, 2009.

48. Asada N and Katayama Y: Regulation of hematopoiesis in endosteal microenvironments. Int J Hematol 99: 679-684, 2014. 